\title{
Performance Evaluation of PAM-4 and PAM-2 Modulation Techniques using MATLAB
}

\author{
Waliu Olalekan, Apena
}

\begin{abstract}
Two Levels Pulse Amplitude Modulation techniques (PAM-2) is failing to cope with the increase in appetite for data and higher data rate with respect to noisy channel. Hence, Four Levels Pulse Amplitude Modulation techniques (PAM-4) was developed. PAM-4 is said to enable two times the data rate as compared to PAM-2. PAM-4 has its pros and cons as compared with PAM-2. This paper compares the performance of Two Levels Pulse Amplitude Modulation technique to Four Levels Pulse Amplitude Modulation technique. The communication system was modeled using Simulink R2016b, the signal passed through an Additive White Gaussian Noise (AWGN) Channel and eye diagram scope was adopted to evaluate the corresponding signal. The study compared eye diagram to evaluate both PAM-2 and PAM-4 at same channel condition. Bit error rate (BER) analysis of the two signals were studied and the results for the corresponding signals were compared. It was observed that the performance of PAM-2 surpasses PAM-4 in terms of the bit error rate analysis. The study shows similar characteristic for Symbol Error Rate Probability curve. The result revealed that PAM-4 is more susceptible to noise than PAM-2.
\end{abstract}

Index Terms-AWGN; BER; Eye; PAM-2 and PAM-4

\section{INTRODUCTION}

Digital signals are transmitted over communication links with respect to nature of the signal (data) and available processing technique(s). The most commonly used modulation techniques for data transfer system are two levels pulse amplitude modulation techniques (PAM-2), this has limitation on data rate (bandwidth) and cost effective [1]. This was addressed with provision of high data rate system called Four Levels Pulse Amplitude Modulation (PAM-4) [1]. A whitepaper presented by Keysight Technologies in DesignCon 2016 states that next generation of OIF and IEEE signaling standards are seriously considering PAM-4 signaling at $56 \mathrm{~Gb} / \mathrm{s}$ over electrical channels [2]. The study investigates effect of noise on Two Levels Pulse Amplitude (PAM-2) modulated signal and Four Levels Pulse Amplitude (PAM-4) modulated signal through an Additive White Gaussian Noise (AWGN) channels. The resulted noisy signals were evaluated using their eye diagram. The system was modelled and simulated in MATLAB SIMULINK R2016b environment. In addition, the bit error rate (BER) of the two signals were analyzed in MATLAB with the aid of plots, comparing both the

Published on May 18, 2018.

Dr. W. O. Apena,

Department of Electrical and Electronic Engineering School of Engineering and Engineering Tecchnology PMB 704, Akure. Nigeria and Biomedical Computing and Engineering Technology, Research Group, Coventry University, UK. (e-mail: woapena@futa.edu.ng; apenaw@coventry.ac.uk). simulated and calculated bit error rates for various signal to noise ratio (SNR) values.

\section{Digital COMMUNICATION SYSTEM}

Communication has to do with transfer of messages or information between two entities. Communication systems usually consist of the source, the channel and the destination or the sink. Digital communication system transmits information in form of binary data. Digital communication could also refer to as data communication [12]. According to [3], the major advantage of digital communication is that signal fidelity is better controlled through digital transmission than analog transmission.

\section{A. Additive White Gaussian Noise Channel (AWGN)}

The Additive White Gaussian Noise (AWGN) Channel is a model of a real communication channel, implementing the effect of noise on the digital signal when passed through a physical communication system. The channel adds a Gaussian distributed noise to the signal when it is passed through it [4].

$$
r(t)=\mathrm{s}(\mathrm{t})+\mathrm{n}(\mathrm{t})
$$

where, $\mathrm{r}(\mathrm{t})$ is received signal, $\mathrm{s}(\mathrm{t})$ is sent signal and $\mathrm{n}(\mathrm{t})$ is the Gaussian distributed noise.

Noise is refers to as distortion and interference in digital communication system, other disturbances that can corrupt a signal include fading, which can be slow fading or fast fading [5]. Also, Jitter pose odds to reference clock signals in electronic link as the deviation from true periodicity of a presumably periodic signal [6].

\section{B. Signaling Techniques}

In order to transmit digital data from the source to destination over the channel, this requires processing techniques such as amplitude and frequency variation. Digital data can be converted to digital signal with the aid of signaling techniques or encoding. The unique examples of processing variation are Two Levels Pulse Amplitude modulation (PAM-2) and the Four Levels Pulse Amplitude Modulation (PAM-4).

\section{1) Two Levels Pulse Amplitude Modulation (PAM-2)}

The common and easiest way of transmitting digital signals is adoption of two different voltage levels for two binary digits [7]. This form of signaling technique is referred to as Two Levels Pulse Amplitude Modulation (PAM-2) as shown in Fig. 1; it could be either Non- Return to Zero (NRZ) nor Return to Zero (RZ). 


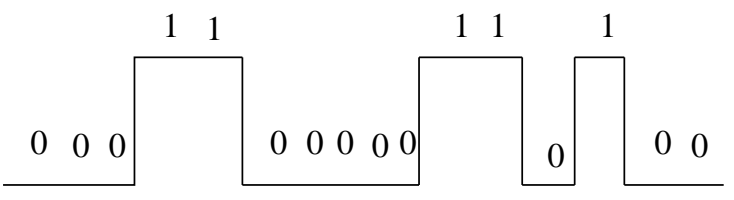

Fig. 1. PAM-2 signal

\section{2) Four Levels Pulse Amplitude Modulation (PAM-4)}

Four Levels Pulse Amplitude Modulation technique (PAM-4) signal processing employed four possible levels. Fig. 2 represents four possible combinations of 2 bits $(00$, 01, 10, 11). Four Levels Pulse Amplitude modulations is militated by bits' data rate and channel for specific transmission media [9].

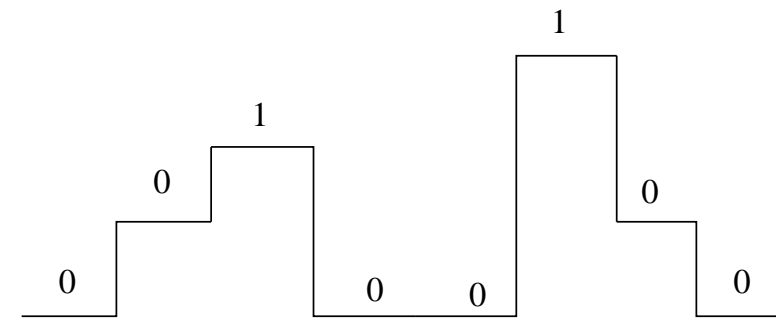

Fig.2. PAM-4 signal

\section{Bit Error Rate (BER)}

Bit error rate (BER) is refers to as the rate at which error(s) occurs in transmission system. According to [11], the bits are subjected to unpredictable changes in the processing such as interference and distortion. Although, knowledge of error detection and correction techniques could be deployed, this is to checkmating BER and hence signals transmission reliability [8].

\section{Eye DiAgram AND THEORETICAL ANALYSIS}

Eye diagram is a useful tool to understand signal impairments in the physical layer of high speed data system [10]. It is adopted tool for evaluation of combined effects in channel noise and interference on transmitted signal and system performance. Eye diagram could support system evaluation to study signal behaviors such as damping, clock synchronization, noise and overshooting. Furthermore, eye diagram can also improve signal knowledge on amplitude, height, crossing time features and width. Eye pattern corresponds to minimal signal interference and distortion(s).

\section{A. Eye Diagram Analysis (PAM-2/PAM-4)}

The SIMULINK analysis of the Eye diagram for a PAM2 signal was developed using a random integer generator (source) and the outputs modulated PAM-2 modulator as shown in Fig. 3. The modulated signal was corrupted in additive white Gaussian noise (AWGN) channel and the output was viewed through an Eye diagram scope. Reverse process was carried out with adoption of raised cosine receive filter while a PAM-2 demodulator was used to inverse the transmitted signal. The signal to noise ratio $\left(\mathrm{E}_{\mathrm{b}} / \mathrm{N}_{\mathrm{o}}\right)$ property of the AWGN channel was also varied to study the effect of noise on the signal.

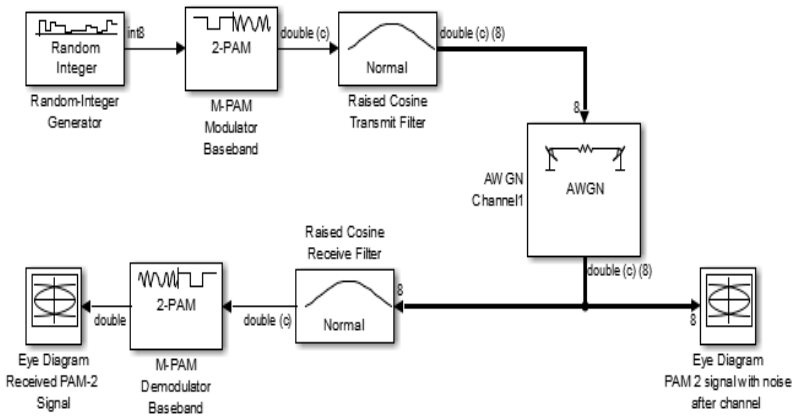

Fig. 3. Simulink Block for PAM-2 System

The same process and analysis was developed for PAM-4 as shown in Fig. 4. This study was carried out for details evaluation between PAM-4signal and PAM-2modulators.

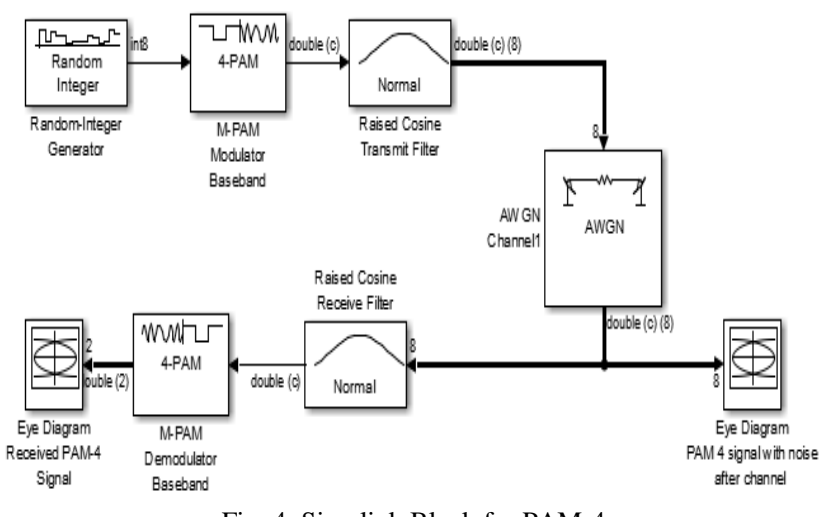

Fig. 4. Simulink Block for PAM-4

\section{B. PAM-2/PAM-4 Bit Error Rate (BER) Theoretical} Analysis

Considering binary PAM baseband signals, with two signal waveforms represented as;

$$
\begin{aligned}
& s 1(t)=g(t) \\
& s 2(t)=-g
\end{aligned}
$$

$\mathrm{s} 1(\mathrm{t})=-\mathrm{s} 2(\mathrm{t})$ are antipodal signal. Where, the energy in the pulse $\mathrm{g}(\mathrm{t})$ is equal to the energy per bit $(\mathrm{Eb})$. Hence;

$$
\begin{aligned}
& s 1=\sqrt{E b} \\
& s 2=-\sqrt{E b}
\end{aligned}
$$

Since the two signals are equal, the received signal after the signals pass through an AWGN channel will be assuming s1 was transmitted will be;

$$
r=s 1+n=E b+n
$$

where, $\mathrm{n}$ represents the additive white Gaussian noise (AWGN) noise component in the communication link.

If $r>0$, the decision is made in favor of $s 1(t)$ and if $r<0$, the decision is made that $s 2(t)$ was transmitted signal. The conditional probability density function (PDF) for the two signals is presented in (7) and (8). 


$$
\begin{aligned}
& P_{b}=\frac{1}{2} P(e \mid s 1)+\frac{1}{2} P\left(\left.e\right|_{s} 2\right) \\
& P\left(\left.e\right|_{s} 1\right)=P(e \mid s 2)=\frac{1}{2} \operatorname{erfc}\left(\frac{Q}{2}\right)
\end{aligned}
$$

where, $\mathrm{Q}$ is also referred to as the signal to noise ratio (SNR) in the system. However, (7) and (8) could be simplified as (9) and (10) respectively.

$$
\begin{aligned}
& P_{b}=\frac{1}{4} \operatorname{erfc}\left(\frac{Q}{2}\right)+\frac{1}{4} \operatorname{erfc}\left(\frac{Q}{2}\right) \\
& P_{b}=\frac{1}{2} \operatorname{erfc}\left(\frac{Q}{2}\right)
\end{aligned}
$$

The value of the signal to noise ratio $\mathrm{Q}$ was varied from 10 to 20 decibel $(\mathrm{dB})$ and plotted values was developed using MATLAB script.

\section{Bit Error Rate Analysis of PAM-2 (Simulation)}

The study validates the behavior of the bit error rate as the signal to noise ratio varies by simulation of bit error rate analysis of PAM-2 signal in MATLAB R2016b environment, through generated binary data passed through a PAM-2 modulator. The resulted signal was passed through an Adaptive White Gaussian Noise (AWGN) Channel of signal to noise ratio (varying from -10 to $20 \mathrm{~dB}$ ). The output of the demodulator was compared to the original binary data using the system object communication Error Rate to derive the error in the received bits.

\section{Bit Error Rate Analysis of PAM-4 (Theoretical)}

The probability of error in a PAM-4 transmitted signal can be derived from the probability of error of PAM-L signal as shown in (11).

$$
P_{e}=\frac{L-1}{L} \operatorname{erfc}\left(\frac{Q}{\sqrt{2(L-1)}}\right)
$$

where, $\mathrm{L}$ is the number of levels; Putting $\mathrm{L}=4$. (11) could be adjusted as written in (12).

$$
P_{e}=\frac{a}{4} \operatorname{erfc}\left(\frac{Q}{a \sqrt{2}}\right)
$$

The value of the signal to noise ratio $\mathrm{Q}$ was varied from 10 to $20 \mathrm{~dB}$ and plotted values was developed using MATLAB script R2016b environment.

\section{E. Bit Error Rate Analysis of PAM-4 (Simulation)}

Similar to PAM-2 signal, the study validates the behavior of the bit error rate (BER) as signal to noise ratio varies. The simulation of PAM-4-bit error rate analysis was carried out in MATLAB R2016b environment. The resulted signal was passed through Adaptive White Gaussian Noise (AWGN) Channel with varied signal to noise ratio varying ( -10 to 20 $\mathrm{dB})$. The resulted signal was demodulated in the output using a PAM-4 demodulator. The study compared the output after demodulation.

\section{RESULTS AND DISCUSSION}

The study considered PAM-2 and PAM-4 performances with respect to signal to noise ratio (SNR). The eye diagram and bit error rate performance were evaluated respectively.

\section{A. E ye Diagram Results: PAM-2 and PAM-4}

PAM-2 signal is passed through an AWGN channel of signal to noise ratio (SNR) of $40 \mathrm{~dB}$ as shown in Fig. 5 . Similarly, PAM-4 signal was investigated under same condition as shown in Fig. 6.
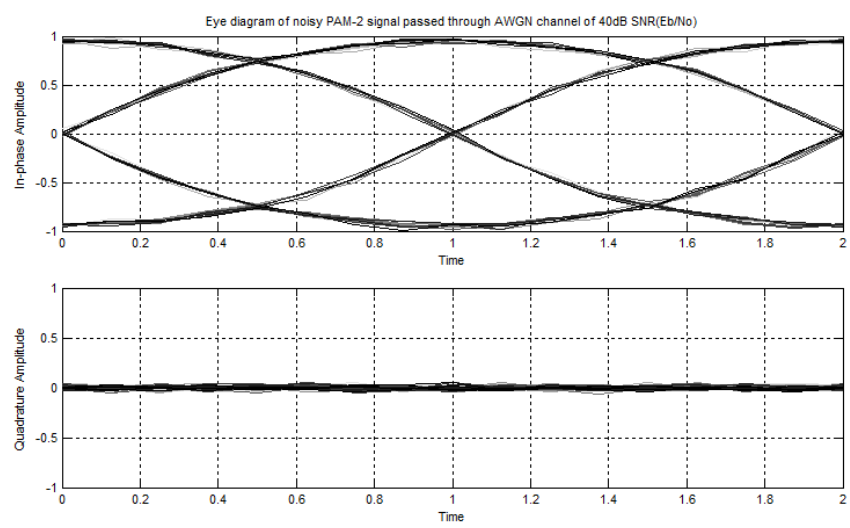

Fig.3. PAM-2 Eye Diagram Against 40dB SNR (Eb/No)

Comparing the eye diagrams in Fig. 5 and 6; these illustrates that the vertical eye opening of PAM-2 signal is vertically wider than PAM-4 signal. However, the PAM-4 could be posed to distortion and interference. Furthermore, the simulation scope reads the vertical eye opening of PAM2 as $1.5 \mathrm{AU}$ while that of PAM-4 is $0.5 \mathrm{AU}$. Consequently, it PAM-4 could requires more complex signal processing techniques to minimized SNR.
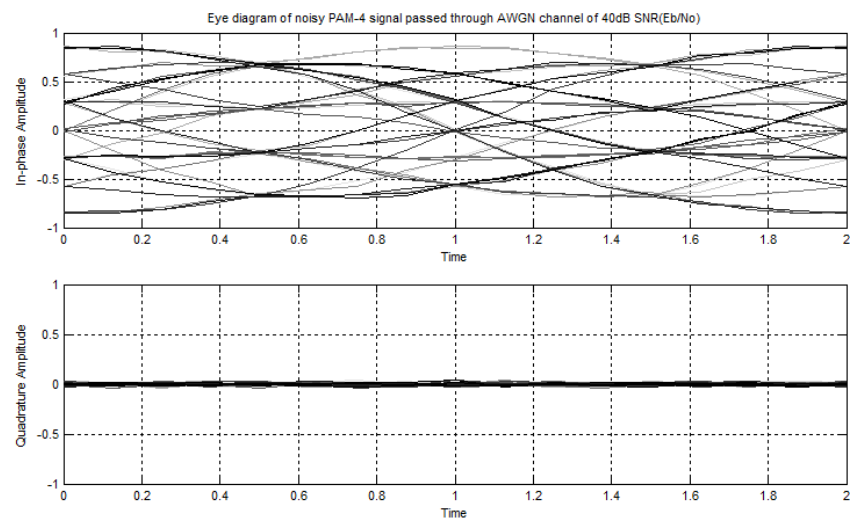

Fig.4. PAM-4 Eye Diagram Against 40dB SNR (Eb/No)

The study investigated a scenario of reduced SNR (25 dB) for both PAM-2 and PAM-4 to signal performance in the channel as shown in Fig. 7 and Fig. 8 respectively. The result revealed distortion in the system as the channel noise reduces compared to $40 \mathrm{~dB}$ above. This shows that PAM-2 could withstand noisy channel and better performance at high SNR. 

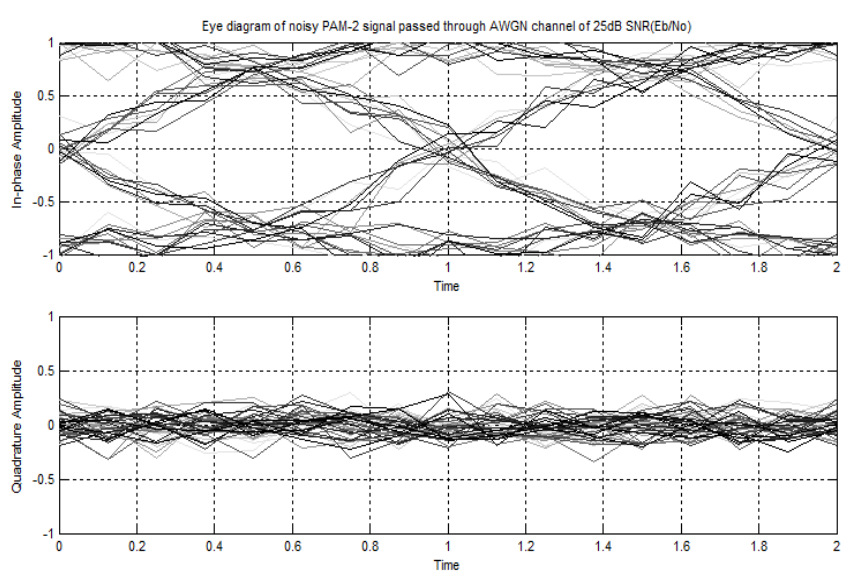

Fig.5. PAM-2 Eye Diagram Against 25dB SNR (Eb/No)

Fig. 8 revealed inability of PAM-4 coping with distortion at $25 \mathrm{~dB}$ noisy channel in comparison to $40 \mathrm{~dB}$ above. However, the result hardly distinguish the eye levels of PAM-4 compared to PAM-2, this indicates that PAM-4 is more susceptible to noise.
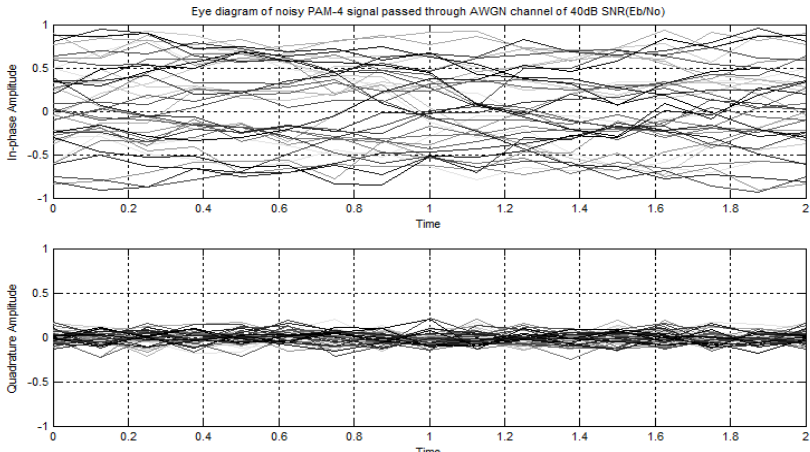

Fig.6. PAM-4 Eye Diagram Against 25dB SNR (Eb/No)

\section{B. Bit Error Rate Analysis Result: PAM-2 and PAM-4}

The theoretical symbol error probability for both PAM-2 and PAM-4 signals are plotted in Fig. 9. The effect of increasing the signal to noise ratio (Es/No) with respect to symbol error rate in revealed in Fig. 9 and Fig. 10. It can be deduced from the Fig. 9 and 10 that PAM-4 is susceptible to noise than PAM-2 at same SNR value.

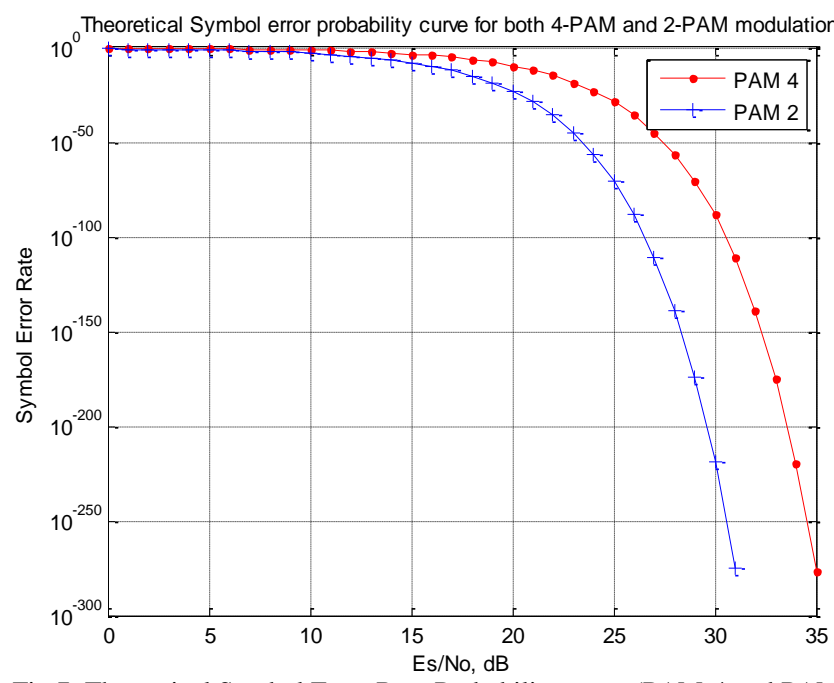

Fig.7. Theoretical Symbol Error Rate Probability curve (PAM-4 and PAM2)
The simulated symbol error probability plotted for both PAM-2 and PAM-4 signals is shown in Fig. 10. The effect of increasing the signal to noise ratio $(\mathrm{Eb} / \mathrm{No})$ with respect to symbol error rate using MATLAB R2016b simulation environment is revealed in Fig. 10. The study shows similarity characteristic for both PAM-2 and PAM-4, as symbol error rate reduces the SNR increases. Also, as the SNR increases the performance of PAM2-2 and PAM-4 seems to be converging.

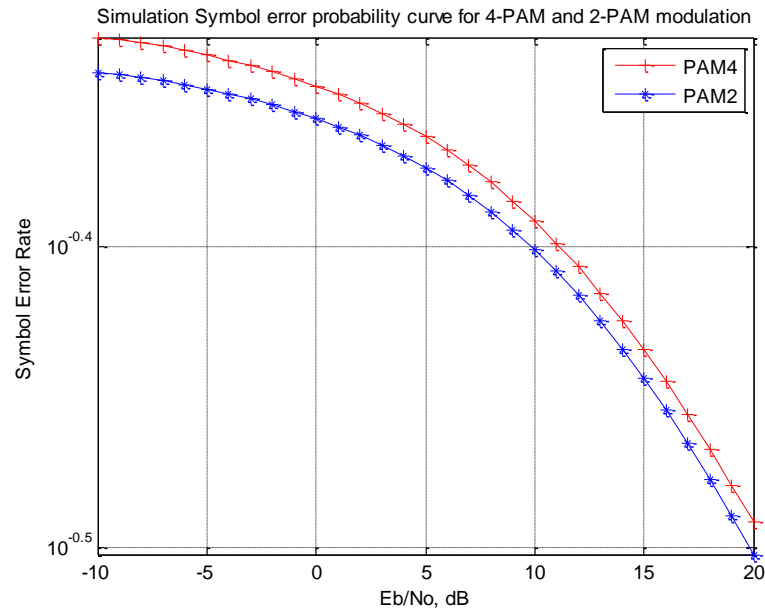

Fig.8. Simulated Symbol Error Rate Probability Curve (PAM-4 and PAM2)

\section{CONCLUSION}

The study has vividly considered PAM-2 and PAM-4 using eye diagram, SNR, BER and Symbol Error Rate Curve as indices of evaluation. The result revealed that PAM-2 could withstand increase in SNR as investigated using $40 \mathrm{~dB}$ and $25 \mathrm{~dB}$ AWGN above. The eye diagram revealed susceptible of PAM-4 to noise. However, SER curve performance of PAM-2 is better than PAM-4. PAM-2 has similar signal bandwidth characteristic to PAM-4 (half) in terms of transmission level. Performance of PAM-2 in terms of bit error rate is better than that of PAM-4 at to the same AWGN channel property pose a better modulation technique for noisy channel above $40 \mathrm{~dB}$.

\section{ACKNOWLEDGMENT}

I acknowledge the support given by my students, Abode Daniel (B.Eng., 2016/2017) and Ali Hafis (M.Eng., 2017/2018) for this study. More grease to your elbows and thank you for your support.

\section{REFERENCES}

[1] Martin R, PAM-4 A New Measurement Science, [Online, 2015] www.ednvault.com/new_measurement_science. www.ednvault.com. [Cited: August 3, 2017.]

[2] Liever D., PAM-4 Simulation to measurement validation with commercially available software and hardware, Keysight Technologies. (2016) 5992-1320EN.

[3] John G.P. and Masoud S. Communication System Engineering Prentice Hall, Upper Saddle River. (2002) ISBN: 0-13-061793-8.

[4] Samson A.O. and Ayoola A.A. Comparing Performances of Bandpass Modulation in Wireless Communication Channels. Researchpub, Akure. Vol. 2. (2013) ISSN: 2165-8315. 
[5] Motchenbacher C.D., and Connelly J.A., Low Noise Electronic Design, John Wiley \& Sons, INC, New York. (1993) ISBN10:0571577421.

[6] Wolver D. H., Phase Locked Loop Circuit Diagram, Prentice Hall, Upper Saddle River. (1991). (ISBN)

[7] William S. Data and Computer Communications, Prentice Hall, Upper Saddle River. (2007). ISBN: 0-13-243310-9

[8] Electronic design, Bit Error Rate Testers face Ethernet Speed Challenges, [Online, 2016] www.electronicdesign.com. [Cited: August 8, 2017].

[9] Tektronix, PAM4 Signaling in High Speed Serial Technology, [Online, 2015. www.tek.com/application/100g-optical-electrical-txrx. ISSN: 55W-60272-0. [Cited: December 20, 2017]

[10] Understanding Eye Pattern Measurements. Anritsu, Texas. Application Note No: (2010) 11210-00533.

[11] Behrouz A.F. and Sophia C.F. Data Communication and Networking, McGraw-Hill, New York. (2007) ISBN-13: 978-0-07-296775-3

[12] Edward A.L. and David G.M., Digital Communication, Kluver Academic Publishers, Massachusetts. (1994) ISBN: 0-7923-9391-0.

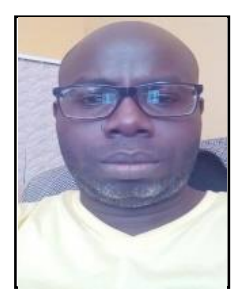

Waliu Olalekan, Apena. - Doctor of Philosophy $(\mathrm{PhD})$ in Biomedical Computing and Engineering Technologies, and Master of Science in ICTs for Engineers (Digital Communications option) from Coventry University, United Kingdom and B.Eng. in Electrical and Electronic Engineering from the Federal University of Technology, Akure, Nigeria. Research interest includes, Digital Communications Technologies, Healthcare Informatics, Biomedical Engineering; Electrical and Electronic Engineering Knowledge Management (EKM) and Wireless Sensor Network (WSN). 